\title{
Cultivating the Therapeutic Alliance in a Telemental Health Setting
}

\author{
Valerie Q. Glass ${ }^{1}\left[\right.$. Aurélia Bickler ${ }^{1}$
}

Accepted: 23 February 2021 / Published online: 11 March 2021

(c) The Author(s), under exclusive licence to Springer Science+Business Media, LLC, part of Springer Nature 2021

\begin{abstract}
Telemental health services have broadened during the last decade (Choi et al. 2019; Pierce et al. 2020). More recently, Severe Acute Respiratory Syndrome Coronavirus 2019 (COVID-19) restrictions have led to an escalation in clinical services through telemental health settings. The purpose of this phenomenological study was to consider perspectives of Marriage and Family Therapists (MFT)s who are working in telemental health settings prior to and/or as a result of COVID-19 restrictions and consider the role that telemental health has in building therapeutic connections with clients. Researchers presented an online survey that explored participants' perceptions of providing telemental health. Participants included 23 MFTs who were currently licensed or working under an MFT supervisor. Data analysis uncovered the following thematic responses: (a) doing telemental health is similar, but different, than in-person therapy, (b) adapting to telemental health is worthwhile, and (c) validating clients' voices and experiences is fundamental to building an alliance in telemental health therapy. Findings supported the importance of further training in telemental health, specifically related to cultural humility and alliance building within telemental health settings.
\end{abstract}

Keywords Therapeutic alliance $\cdot$ Telemental health

\section{Introduction}

\section{Background}

Telemental health services were growing prior to Covid-19 and have expanded exponentially since Severe Acute Respiratory Syndrome Coronavirus 2019 (COVID-19) stay at home restrictions. In the past 5 years, it is estimated that between $5-21 \%$ of mental health services delivered telemental health methods of engagement (Choi et al. 2019; Pierce et al. 2020). With advancements in technology and training over the last few years, some Marriage and Family Therapists (MFT) have been adding telemental health options to their own practices. Since COVID-19, almost all practicing mental health providers have had to adjust their work with clients to include telemental health options (Madigan et al. 2020). This shift, during COVID-19, may prompt many

Valerie Q. Glass

VGlass@ncu.edu

Aurélia Bickler

ABickler@ncu.edu therapists to consider the impact telemental health has on clinical outcomes.

\section{Effectiveness of Telemental Health}

Telemental health services can be an effective option for clients and therapists for several reasons: it is cost effective, it is not limiting due to transportation issues, those in rural areas are more reachable, scheduling can be easier for those with work and family demands, technology has evolved to provide secure platforms for engaging in confidential services, and services can reach people who may not feel comfortable in a traditional setting (e.g., because of mental health challenges, ability differences, or life circumstances) (Langarizadeh et al. 2017; Springer et al. 2020). Research indicates that some clients may seek out telemental health services when issues are more sensitive. In one study, couples addressed more difficult topics (e.g., infidelity) more frequently in telemental health settings, when compared to a sample of couples in an in-person setting (Roddy et al. 2019). This suggests that telemental health services might offer a physical distance that makes is easier to process more sensitive discussions.

$1 \quad 11355$ N. Torrey Pines Rd., La Jolla, CA 92037, USA 


\section{Barriers to Telemental Health}

Some barriers to telemental health care have been researched. These barriers include: feeling like the service lacked a personal connection, difficulty in picking up on non-verbal cues, clinicians' sense that they could not use body language to comfort, inability to connect via eyecontact, challenges with technology or interruptions, limited clinical training or experience with telemental health, limited ability to develop therapeutic rapport, controlling online environments, and privacy or confidentiality (Akyil et al. 2017; Cowan et al. 2019; Wrape and McGinn 2019). Research identifies therapeutic movements into different stages of therapy can be altered by telemental health therapies. Springer et al. (2020) found that spontaneity was more difficult for therapists in telemental health settings. Additionally, working with couple or family conflicts in the moment can feel limiting because of the therapists' use of physical space in difficult situations (Springer et al. 2020).

\section{Therapeutic Alliance in Telemental Health}

The therapeutic alliance is a notable challenge or perceived challenge within telemental health settings (Springer et al. 2020). The therapeutic alliance is crucial to the change process (Fife et al. 2019). Prior to doing telemental health, therapists assumed alliance building would be challenging in a telemental health setting (Springer et al. 2020). Through some clinician adaptations, therapists were able to develop strong therapeutic alliance with telemental health clients. One took that therapists used to increase this strong sense of a therapeutic alliance in telemental health setting was to be more in-tune with "verbal, nonverbal, auditory, and visual cues" (Springer et al. 2020, p. 211). Other ways therapists adapted were to include specific displays of empathy or a "positive regard" for patients (Iankieva et al. 2016). This focus on the therapeutic alliance in telemental health settings is critical in part because research indicates clients tend to feel more comfortable and vulnerable in telemental health settings (Langarizadeh et al. 2017).

One critical element of therapeutic alliance building is the understanding self. This location of self requires an attunement to cultural humility (Perkins et al. 2019). Locating this sense of cultural humility when using telemental health methods is crucial to meeting client needs and establishing a therapeutic alliance (Stevenson 2014; Akyil et al. 2017). Acknowledging one's biases allows for a more authentic, critical and sensitive approach (Watson 2019). Providing space for inclusion, cultural awareness, and the understanding of self and social location creates a bridge for connection between client and therapist (D'Arrigo et al. 2017; Watson 2019).

As clients continue to become more culturally and otherwise diverse (AAMFT 2014), marriage and family therapists are faced with the need to embrace cultural diversity as something they "are" instead of something they "do" (D'Aniello et al. 2016). While some believe that certain models are more innately culturally sensitive than others (Cheung and Chan 2002), some argue that it is an additional component of common factors and is reflected in models across the field as it is essential to the effectiveness of therapy (Cheung and Chan 2002; D'Aniello et al. 2016). Establishing rapport with a client for example, is heavily reliant on the therapist's cultural sensitivity (D'Aniello et al. 2016). A client is only as comfortable to share his or her journey as the therapist allows and promotes in the therapeutic space. This places additional responsibility on all humans to embrace the multicultural and complex riches that our society offers in defining our combined identity (McGoldrick and Hardy 2019).

Bringing cultural humility to telemental health settings is not widely researched (Akyll et al. 2017; Martin et al. 2020). Technology is one way to reach underserved populations and to embrace communication using technology (Akyıl et al. 2017). There is an evolution in how people are communicating within virtual spaces and adapting to this new "virtual language" that exists within telemental health settings. Additionally, telemental health may generate a slightly different clientle, for example, there may be any number of reasons that some clients were not coming to therapy until telemental health options were available. Being competent in cultural humility and the increased intersections of identities that might occur within telemental health settings (e.g., rural, SES, ability, etc.) is crucial to providing quality care and building therapeutic alliances (Martin et al. 2020).

\section{Research Question}

The purpose of this qualitative research was to explore the ways in which MFTs adapted to telemental health services during the COVID-19 health crisis; specifically in relation to therapeutic alliance building. We hoped to better understand the shifts that therapists faced utilizing telemental health and how these experiences could inform future work in telemental health settings. The research question posed in this study was: How do MFTs adapt to telemental health environments, in particular, how do they build connectivity with clients? 


\section{Methods}

\section{Study Design}

This study utilized a phenomenological design (Moustakas 1994; Chong 2019). Qualitative data were analyzed with attention to the phenomenon. Phenomenological research involves researchers stepping back and viewing the data from multiple perspectives (Moustakas 1994; Ahmed 2006; Chong 2019). It involves researcher awareness and a deep sense of listening to what the participants are sharing. Meaning is uncovered through researcher engagement with data (Moustakas 1994). In a final stage of analysis the phenomena of the participants' experiences are identified.

\section{Participants}

Participants included 23 currently practicing MFTs (licensed or working under supervision) throughout the United States. Participants reported working in a variety of settings (e.g., private practice, community services, school-based) with various populations (e.g., teens, adults, couples, families).
A few participants noted specializations (e.g., trauma, substance abuse, LGBTQ+, military families). A majority of participants $(n=12)$ reported working as an MFT for over 10 years; 7-10 years $(n=3)$; 4-6 years $(n=3)$; $1-3$ years $(n=4)$, unreported $(n=1)$. About half of the participants $(n=12)$ reported doing telemental health prior to COVID-19; while the other participants $(n=11)$ have only been doing telemental health since COVID-19 restrictions. Of the participants, $n=18$ identified their race as White; $n=3$ as Black or African American, $n=1$ as mixed race, and $n=1$ was unreported. Twenty-one $(n=21)$ of the participants identified themselves as "female" or "cisgender female"; one participant $(n=1)$ identified himself as male; and one left gender unreported. Participants were given pseudonyms to ensure confidentiality. Demographic information can be found in Table 1.

\section{Recruitment}

Prior to recruitment, researchers obtained approval through the Internal Review Board of their university. Permission to pursue the research project, including identifying and recruiting human subjects, was granted. Recruitment for the study included respondent-driven snowball sampling

Table 1 Participant Demographics

\begin{tabular}{|c|c|c|c|c|c|}
\hline Pseudonym & Gender & Race & State & Years of Experience & $\begin{array}{l}\text { Telehealth experience } \\
\text { prior to Covid-19 restric- } \\
\text { tions }\end{array}$ \\
\hline Amy & Female & White & MI & $>10$ years & Yes \\
\hline Benita & Female & White/Hispanic & $\mathrm{FL}$ & 4-6 years & No \\
\hline Carly & Female & White & $\mathrm{MN}$ & $>10$ years & Yes \\
\hline Dina & Female & White & $\mathrm{TX}$ & 4-6 years & No \\
\hline Emily & Female & White & $\mathrm{TX}$ & $>10$ years & Yes \\
\hline Francine & Female & White & $\mathrm{CA}$ & $7-10$ years & No \\
\hline Gail & Female & Black & GA & 4-6 years & Yes \\
\hline Haley & No answer & No answer & No answer & $>10$ years & No \\
\hline Inca & Female & Mixed race (Black, White, Hispanic) & FL & $7-10$ years & Yes \\
\hline Jacqueline & Female & White & FL & $>10$ years & Yes \\
\hline Kendra & Female & White & No answer & $>10$ years & Yes \\
\hline Leonard & Male & White & VA & $1-3$ years & No \\
\hline Molly & Female & White & OR & $>10$ years & No \\
\hline Nina & Female & White & FL & $7-10$ years & Yes \\
\hline Olivia & Female & Black & VA & $1-3$ years & No \\
\hline Patricia & Female & White & MA & $>10$ years & No \\
\hline Quiana & Female & Black/African American & MO & No answer & No answer \\
\hline Rachel & Female & White & $\mathrm{CA}$ & $>10$ years & Yes \\
\hline Samantha & Female & White & $\mathrm{KS}$ & $>10$ years & Yes \\
\hline Tilly & Female & White & VA & $1-3$ years & Yes \\
\hline Uri & Female & White & MI & $>10$ years & Yes \\
\hline Victoria & Female & White & No answer & No answer & No \\
\hline Winnie & Female & White & No answer & No answer & No \\
\hline
\end{tabular}


(Heckathorn 1997; Baltar and Brunet 2012). Participants self-selected to participate in the study from a social media posting. Researchers posted recruitment information to personal and professional social media sites. Recruitment materials indicated that the study intended to research MFTs utilizing telemental health methods prior to or as a result of COVID-19. The recruitment script encouraged participants to pass the survey along to colleagues. Criteria to participate included: (a) currently licensed as an MFT or working under the supervision of a licensed MFT, (b) being clinically active at time of survey, and (c) having had experience in telemental health platforms.

\section{Survey Instrument}

After agreeing to participate, participants had access to a survey tool that they completed online on their own. Survey questions included a few demographic questions and openended questions regarding telemental health experiences. Questions explored participants experience with telemental health, their visions of the pros and cons of telemental health, how they feel they adapted to telemental health, and their experiences with connectivity and alliance building within their telemental health work. Some examples of survey questions included:

1. Tell me a little about what the adjustment to telehealth was like for you.

2. Are you noticing any specific clinical themes related to COVID-19 in your practice and, if so, what are you seeing that might be different that in-person therapy?

3. Can you identify any tools or techniques that you utilize in the telemental health setting?

4. Tell me a little about the differences that you are seeing related to building a therapeutic alliance between inperson and telemental health?

5. Do you feel like you and your clients make similar progress and meet goals similarly in telemental health and in-person settings?

6. Do you have any tools or techniques for working specifically with couples and families in telemental health settings?

7. What is one thing that you bring to your work that makes you unique and you feel makes a difference to the change process with clients and does that differ in the online setting?

\section{Data Analysis}

Coding and analysis of research data derived from the Analytic Guiding Frame (AGF) and Overall Guiding Frame (OGF) identified by Chong (2019). These frames identify that "...the researcher uses an interpretivist or constructivist perspective to trace a basic pattern of how a phenomenon is experienced across a group of participants" (Chong 2019, p.301). The process of the AGF includes: engaging with the data initially through a reflexive lens, looking at the data from various perspectives and creating "critical connections" (p. 300) with the data. The four stages of this analysis process include:

1. Setting up the research process and analysis.

2. Initial data analysis which includes focused attention on the data and the emergence of themes.

3. Categorizing data into "meaningful units" (p. 301).

4. Bringing together units and themes with research questions and previous research to determine major findings.

According to the OGF framework, the analysis requires researchers to be mindful of two specific questions throughout the analysis process: (a) "How does this data illuminate my research objective?" and (b) "How does this data raise new questions about my research objective?" (Chong 2019, p.302). The AGF and OGF frameworks fits nicely with the phenomenological design because it allows the researcher to constantly be in tune with the participants and their context while checking thematic codes with previous research. Additionally, this framework nicely connects the themes within the data in a way that allows for cultivation of phenomena (Chong 2019).

\section{Critical Reflexivity}

Phenomenological research recognizes the integration of researcher perspectives (Moustakas 1994; Chong 2019). During the data collection and analysis, we (the researchers) remained focused and aware of our own perspectives and possible biases as we engaged with the data. Both researchers involved in the analysis are practicing MFTs with a focus on cultural humility and social justice. We both teach and do clinical work in virtual settings and have our own perspectives on the strengths and challenges of in-person and virtual educational and clinical work. Prior to COVID-19, we both practiced clinical work in both in-person and virtual settings.

\section{Results}

The purpose of this research study was to explore MFTs experiences building connections with clients in a telemental health setting. We were most interested in understanding some of the ways MFTs have adapted their telemental health practices to include building the therapeutic alliance. Surveys were completed by 23 participants. The phenomena of participant experiences fell into three major themes: (a) doing telemental health is similar, but different, than 
in-person therapy, (b) adapting to telemental health is worthwhile, and (c) validating clients' voices and experiences is fundamental to building connectivity in telemental health therapy.

\section{Theme \#1: Doing Telemental health is "Similar," but Different, than In-person Therapy}

Doing telemental health therapy with individuals, couples, and families often encompasses shifting into a new paradigm. Participants shared experiences of working in telemental health that allowed for building a therapeutic alliance, goal setting, the change process, and modality application. Coded themes revealed a phenomenon that typical therapeutic processes were present and strong in the telemental health settings, they just required a different lens, a different understanding of the process. The data represented a view of telemental health as not "comparing" to in-person therapy but as having its' own distinct process. For example, Gail shared, "Energy and body language are evident in-person. Comfort and freedom of expression are evident during telemental health." She does not indicate that she is comparing the two, just noting the strengths in each of the processes.

Themes evolved into meaningful units that addressed strengths. A notable strength that emerged from these unit included client vulnerability in telemental health settings. The phenomenon present implied that participants mentioned that clients were able to present vulnerable situations that seemed different than what the therapists experienced in in-person settings, there was a deeper sense of safety. Quiana stated, "some people have more courage when able to sit behind a screen." Molly shared "some of my clients have opened up about deeper trauma and wanted to work through it...the virtual setting creates less vulnerability, therefor, (they are) willing to open those doors." Haley stated, "They can share more of themselves just being in their homes. And are more willing to take emotional risks." Therapists attribute this sense of vulnerability to clients being in their own environment and also to the sense of safety that a "screen" between the client and therapist can present.

In connecting the research to our analysis, research on rapport building in a telemental health setting suggests that therapists are cautious about the ability to build a strong therapeutic alliance in a virtual setting (Fife et al. 2019). Participants were asked directly about their ability to build a therapeutic alliance in a telemental health setting and most $(n=18)$ reported strong alliances (just as strong if not stronger) than they experienced in in-person settings. Four participants $(n=4)$ felt that rapport was better in the in-person setting, and one $(n=1)$ did not report.

Alliance building in the telemental health format allows therapists to enter the client's visual world. The data uncovered a meaningful unit around how therapists engaged with participants' home environments. Participants noted being able to see the background of client homes and comment on elements of clients' environments that allowed for deepening of the therapeutic alliance. Therapists thought that access to the family system also provided an additional rapport building strength. Having connections to clients' surroundings allowed therapists to visualize, at times, how clients interacted with the systems in their lives. Additionally, other family members could be part of the therapeutic process that might not have been available otherwise. It was also useful for therapists to see clients in their setting and connect with this. Amy stated, "I think being in the client's environment is really connecting, seeing their families, their pets, the décor, etc." Carly had similar thoughts "(I) can see their home environment, see them interact with pets or family at home... without makeup or façade of public norms of how they should look...(there is) an ease of talking conversationally with less restriction." This sense of comfort brings with it a smooth ease into building the therapeutic alliance

Connecting to the family system was a notable phenomenological strength of telemental health. Inca's response reflected this theme. She saw benefits of clients being in their own context in a variety of ways. She noted that seeing the clients in their environment contributed to her connection to the client and her understanding of where they were coming from. From a systemic perspective, she could meet clinical goals by utilizing the system. At one point Inca shared about building rapport in the virtual setting, "I feel like during telemental health, my psychosocials are less structured and that I do more self-disclosure in joining with the client. For example, if a poster is visible in their background of something I am interested in, I'll share that and we join over these things." She stated later on that there is "flexibility in family therapy sessions. (I) can easily include or remove members from the session as clinically indicated, allowing me to get a lot more done in one sessions that in person." Inca found that having the client in their family/ home setting during session not only allowed her access to the system to build a stronger alliance, but it also allowed her to build in the aspects of the client's environment (e.g., the family system) in a way that created change during the session.

Creating the therapeutic alliance in the telemental health setting, maintaining that alliance, and moving into the change process required an intentional focus on tone, language, and eye contact. Participants often compared the use of body language in in-person work with the shift to focusing on other visual and auditory cues and positioning in the telemental health environment. It was as if there was a refocusing that they had to attend to in the telemental health setting. For example, Jacqueline found that she tended "to rely more on verbal comfort, as opposed to providing tissues, 
a comforting hand, or leaning toward (her) client." Knowing that the connection of physical space and the "space between" (as Amy put it) was not there in the same way as it was in in-person settings, therapists utilized different skills to create this sense of empathy and compassion. Uri stated, "online, you can still communicate empathy with an increased focus on tone of voice". Dina shared "I am tuned into the client's eyes and changes in voice". Rachel shared "in telemental health, you can see more detailed facial expressions". Participants frequently mentioned that their awareness of their own tone and the clients, as well as the focus on facial expressions and eye contact were unique ways they were able to build on the therapeutic relationship in the telemental health setting.

Participants echoed the value of accessibility in telemental health settings. Accessibility was highly regarded in some way by all participants. Accessibility included clients being more comfortable in their own settings due to mental health or physical health barriers, clients who could make telemental health sessions more easily due to time demands or childcare issues, clients that prefer the convenience (e.g., less travel time, scheduling flexibility), and meeting the needs of rural clients who may not have sought services otherwise. Tilly shared, "some clients have told me how they prefer the telemental health setting and how it has increased their willingness and ability to come to therapy." Leonard stated, "For some parents telemental health has been helpful to chat with me during the day while kids are in childcare or after bedtime." Quiana shared that she can reach clients with "mobility issues, transportation issues, rural clients, those who can't leave home, and single parents." She further explains telemental health has "opened up the option for therapy" for people who could not utilize services before. Similarly, participants discussed how telemental health allowed them "access to family members" (as stated by Francine) who might be unable to make in-person sessions for similar reasons (e.g., mobility issues, scheduling issues, etc.). Overall, there is a sense that telemental health can reach previously unreachable clients and family members and that the scheduling conveniences are helping retain clients.

\section{Theme \#2: Adapting to Telemental health is Worthwhile}

Data reflected that adapting to the telemental health formats felt a bit daunting at first to many participants, particularly the ones that were "forced" at the last minute to move to virtual options because of COVID-19 restrictions. Even given these initial concerns, most (all but two) respondents noted they felt pleased in the transition and found the ease of the switch to telemental health was more rewarding than they would have anticipated. Kendra summed it up nicely, she stated, "It is an honor and privilege to do this work each and every day, especially with a population who might not have reached out if it wasn't for this option of telemental health, it breaks down the walls of the walled-off, so to speak." Dina stated that "initially (it was) very stressful" to adapt to telemental health, but in time, she stated, "now, I really enjoy it." Tilly stated, "At first it was hard because of the unknown factors and the speed at which things changed, and simply adapting to a new normal, at this point I feel comfortable with telehealth". Quiana reaffirmed these statements, stating, "(it was) a struggle at first, but (I am) no longer resistant." Molly also added that clients were able to shift to telemental health fairly seamlessly, she stated "it was challenging at first and then everyone settled in."

It is notable that adapting was a phenomenon that was prevalent in participants' responses and most frequently this was viewed in a favorable light. This adapting did not go without some challenges. Participants did note some technology issues, some issues with interruptions, and some issues with engagement. Leonard shared some of his difficulties "it was difficult to get clients to engage, particularly kiddos under 8." Other participants stated that they struggled with clients having access to technology and being challenged with technology. Even with adaptability, at times, a couple of therapists seemed to feel that the body language and rapport building may not have met their expectations.

\section{Integrating self}

A notable sub-theme for therapists was the benefits that telemental health had brought to their personal life. For example, Dina shared, "I am able to be more thoughtful and feel more rested during the day when seeing multiple clients. I am able to take a break in the comfort of my own home instead of being at the office for 10 hours." Haley shared, "I can have more availability to my kids" while working in a telemental health setting, I can "pop in on my family in between sessions." There was a sense that, for therapists, being in a telemental health setting brought them convenience and a better work/life balance.

Therapist participants in this research study were asked about their own strengths as a therapist. Participants' words felt proud, confident, and passionate when they described their own personal strengths as a therapist. These words feel particularly important as they represent an empowered and compassionate MFT field. We felt it was valuable to highlight these strengths in the participants' own words; these words highlight the personal strengths that they brought to building rapport with clients (specifically in a telemental health setting) and allowing for cultural humility. The language around this question included some of these responses: 
- "I seek to show them that I am their teammate on their journey." (Benita)

- "Knowing that we are together doing it." (Carly)

- "Processing and normalizing all of the difficult and raw emotions." (Dina)

- "Humor! And using non-assuming language." (Francine)

- "I am compassionate, yet firm." (Gail)

- "I focus on building a relationship with clients based on respect and meeting them exactly where they are, not where anyone thinks they should be." (Inca)

- "I provide a mixture of humor, self-disclosure, transparency, and collaboration." (Uri)

- "Building trust with (my) clients, trusting the process, trusting myself as a therapist, knowing I am there with them to take the deep plunge into unknown emotional territory." (Winnie)

Therapists provided insights into how they do what they do and how they do it well. They were confident in these parts of self that created strong therapeutic shifts for clients. Therapists were asked directly after this question if this part of their professional work differed as they shifted to online settings. Haley's response represents a common theme among clinicians. In the initial question about her own uniqueness as a therapist, she responded, "I am direct and I swear, with permission. Clients report this to be relieving and honest." She presents her "self" and clients seem to appreciate her transparency of self. When asked if this part of her "self" was different in the telemental health setting, she stated, "I am proud of it!" (her unique skills as a therapist) "and still the same with telehealth." Uri's response was "not everything is the same, it is a part of who I am and I can bring that to any situation or setting. That comes with me." Therapists did not seem to struggle presenting their strengths, their best therapist skills, to the telemental health format. This part did not seem to require much adaptation.

\section{Tools and Techniques}

Participants offered perspectives on providing quality therapy using telemental health. Voice, tone, focus on facial expressions, eye contact, and connecting with clients backgrounds and surroundings were mentioned as rapport building techniques previously. Some participants mentioned structure and organization as useful tools for providing telemental health services. This included setting specific goals for sessions and planning for sessions ahead of time, Leonard shared "always have a plan!" and Tilly echoed these words "I think telemental health necessitates more structure." Others shared their own understanding of technology and being able to help clients troubleshoot technology issues was a useful tool. Carly stated that a therapist working in telemental health should have "good knowledge of technology troubleshooting and high bandwidth secure internet." Some participants indicated that normalizing the technology issues, or the telemental health formatting, can aid in clients' comfort. Being aware of one's own environment can also play a role. Dina described this as "online/ video chat etiquette" and shared making sure "lighting is on the face (not from behind), that the face is in the middle of the screen, that you are not using head phones, that you have an appropriate clean background." Uri added to her environment by making sure there was a sense of inclusivity that welcomes all clients, she stated that she "provided cues to (her) inclusivity and culture attunement (pamphlets, pride flags, etc)." There was a focus on communicating the emotions by asking direct questions about what the client was feeling, what their body language was doing, or sharing what their own body language or emotions were doing (as the therapist). Winnie stated, "explain more of your (own) body language and emotions" to clients. In general, participants provided more time and patience in some instances and worked to make sure their background (environment) and their personal attunement could be translatable with regard to the messages they wanted clients to see and hear.

\section{Theme \#3: Validating Clients' Voices and Experiences is Fundamental to Building Connectivity in Telemental Health Therapies}

The term "validate" was repeatedly reflected in the data. Therapists shared the importance of validating clients' challenges with or resistance to telemental health, as a way to build rapport within the telemental health setting, and as a way to connect to clients around sensitive topics.

Benita responded to a question about how she builds a therapeutic alliance with clients with the phrase "VALIDATE, VALIDATE, VALIDATE!!" She followed up with a discussion on how this played out in the therapy room when emotional topics were uncovered. She shared that she approached the topics "very gently and subtly, over-validating and then asked permission for them to share more." This focused on validation provided a safe space in the telemental health setting.

During the time of this research, there were two difficult historical challenges that clients were experiencing. These challenges included isolation, fatigue, unknown future around the virus, and life shifts that were occurring in the midst of the COVID-19 pandemic. The other was the intensity of social climates in response to George Floyd's death while in police custody. The experiences of clients and the therapist's role in working with emotions around these events was evident in participant responses. These challenges presented a deeper and more emotional backdrop to client experiences. Therapists' responses to these difficult emotional experiences highlighted the therapist role 
in validation and the importance of validating these difficult experiences. Dina responded to her own role in helping clients cope with COVID-19 restrictions by "validating exactly how difficult this situation is, being raw with the emotions, and normalizing their feelings and experiences." Jacqueline echoed a similar response to working with clients during COVID-19, "Validation of feelings and experiences, focusing on things within their control." Kendra validated client's experiences with COVID-19 by normalizing through psychoeducation. She helped clients with "grounding tools to decrease trauma reactivity" and educated them on "survival mode (fight, flight, freeze, fawn)." Others validated experiences around the isolation and anxiety by providing self-care guidance.

Validation was a clear theme in how participants discussed current racial climates. Therapists provided space for conversations, listened, and validated. Haley stated, "people of different races are ready to talk, all it takes is an invitation." Carly shared her role during this time was "listening, naming my whiteness, asking their needs, or giving them permission to call out my bias." Benita shared that she provided "a continuous check-in" and "asking them outright how they have been impacted." Therapists provided space and opened up discussions in the room with clients thus validating their experiences and emotions around the difficult climate.

Participants were asked to reflect on ways that they address power imbalances, inequities, and how they present an atmosphere of non-judgement in telemental health settings. Validation of the client's experiences, as well as validation and awareness of self were part of the process of cultural humility. Amy stated, "I am open to clients and will share my own blinders. I typically open up conversation about race, culture, religion, differences of any kind and allow the client to share their perspectives. I am engaged and open to where those conversations take me." Winnie shared a similar perspective, she stated that when working with difference she gives them "an opportunity to discuss their emotions and not have a bias or judgmental way of leading questions or feelings" while also providing "space to explore the good and bad parts of self that come up for them when discussing raw topics." Therapists focused on "curiosity," "reflective listening", "compassion," and "transparency" as ways to present a validating space for welcoming differences.

\section{Discussion}

Building clinical skills within telemental health environments brings with it specific challenges and strengths. Clinicians are often resistant to the perceived challenges that telemental health can bring, most notably, the fear of not building a connection to clients as easily as in in-person settings; however, there seems to be a shift in this resistance with most clinicians who embrace telemental health fairly quickly, after some experience with it (Akyll et al. 2017; Springer et al. 2020). The advantages that some clients and therapists experience within the telemental health setting (e.g., convenience, comfort) provide something unique that is not easily comparable to in-person settings. It is not to say that in-person settings do not also offer particular benefits, it is just that the environments are different.

Given that telemental health is unique, specific training on telemental health would benefit therapists and are currently not consistently part of MFT programs and training (Pickens et al. 2020). Pickens et al. (2020) found that "the effectiveness of telemental health requires unique skills" ( $p$. 186). Training could alleviate some of the fears and resistance that therapists might experience when considering telemental health options. The phenomena of participant experiences in this study lead us to infer some telemental health training topics that would be useful. These would include: understanding technology and platforms, building a therapeutic alliance, translating modality or clinical strengths to telemental health, presenting and reading of facial expression and tone, and embodying cultural humility.

Data support a sense of client vulnerability within telemental health settings. Clients are seeking out telemental health therapies specifically, at times, when their challenges are particularly difficult and they feel a deeper sense of safety with their vulnerabilities in a distanced format (Roddy et al. 2019). Additionally, vulnerable populations are more easily accessible in telemental health setting (Langarizadeh et al. 2017). The increased sense of vulnerability within a telemental health settings and the possible expanded prospect of unique populations could influence clinical directions and alliance building in telemental health settings. Training and cultural competency should rise to meet these specific needs.

Building a therapeutic alliance and the constant attention to that alliance is one of the key components of the change process (Perkins et al. 2019). The therapeutic alliance is generated and maintained through an integration of the therapist's personality, their attention to the clients, and their clinical experience. In telemental health settings, therapists utilize these same variables as they engage in the rapport building process; however, at times, they do it differently. The indicators for alliance building are different in a telemental health setting. Therapists are focusing more on the clients' environments, their tone, and their facial expressions to gauge the strength of the alliance. Additionally, therapists are presenting their own clinical flare through focus on their words, tone, and facial cues. The therapeutic alliance in the telemental health setting includes visuals that tell a story. This differs from in-person settings where the alliance revolves around "telling" of the story, which likely does not 
include the visual background. Therapists can bond with clients over pictures, posters, children in the background, pets, musical instruments, and family members that are part of the clients' worlds. Therapists can get a visual picture of the vibes of clients' homes and get access to significant people in clients' lives. This visual awareness can increase the therapist's understanding of the clients, their presenting problems, and, ultimately, the therapeutic alliance.

\section{Limitations}

The phenomenological approach takes into account the experiences of these 23 participants, who were largely homogenous; 17 of the 23 participants identified as white and women; one as a white male; 3 as African American women; 1 as a biracial woman; and one unknown. This likely presented some similarity in responses and experiences based solely on race.

Limitations could have been present within this research study and analysis with regards to the researchers' professional identities. Both researchers involved in this study have backgrounds working in online clinical environments. Researchers engaged in frequent discussions around the data in order to hone in on themes relevant to participant experiences and research rather than their own assumptions or projections in order to minimize this limitation (Moustakas 1994). Additionally, the researchers sought out participants through colleagues and personal connections, even though the snowball sampling procedure expanded from initial contacts, it is possible some participants' responses reflect similar backgrounds or clinical experiences.

\section{Directions for Future Research}

Research questions within this study asked about cultural humility and discussing challenging political climates with marginalized populations. Participants reflected on being open to difficult conversations and providing a platform for clients to express emotions around topics related to inequities or marginalizations. We felt there was something missing from this data that could better help therapists when considering cultural humility. There was an awareness and an openness to client experiences; however, there was not a focus on sitting with these difficult topics or what could be said to open the door to these conversations. Future research could connect some of these experiences; particularly as they present themselves in a telemental health format. Additionally, future research on training needs specific to working in a telemental health setting would benefit therapists. As the climate around utilizing telemental health shifts, being able to provide adequate training to meet the needs of this platform facilitates a higher standard of care clients receiving telemental health therapy services.

The intensity of COVID-19 was prevalent in this research. Community trauma and the influence of trauma on family systems play a role in daily experiences and resiliency (Landau et al. 2008). Bringing in community connections and family support play a role in coping. Research identifying particular emotions around COVID-19, the trauma influence, and tools for MFTs in the wake of this current traumatic climate, particularly when utilizing telemental health, could benefit the MFT profession.

\section{References}

Ahmed, S. (2006). Queer phenomenology: Orientations, objects, others. Duke University Press.

Akyıl, Y., Bacigalupe, G., \& Üstünel, A. Ö. (2017). Emerging technologies and family: A cross-national study of family clinicians' views. Journal of Family Psychotherapy, 28(2), 99-117. https:// doi.org/10.1080/08975353.2017.1285654

American Association for Marriage and Family Therapy (2014). Accreditation standards graduate and postgraduate marriage and family therapy training programs: Version $12.0 \mathrm{Draft}$. http://www.aamft.org/imis15/Documents/COAMFTE/COAMF TE_Proposed_Accreditation_Standards_Version_12_SecondDraf t.pdf

Baltar, F., \& Brunet, I. (2012). Social research 2.0: Virtual snowball sampling method using Facebook. Internet Research, 22(1), 57-74. https://doi.org/10.1108/10662241211199960

Choi, S., Wilcock, A. D., Busch, A. B., Huskamp, H. A., Uscher-Pines, L., Shi, Z., \& Mehrotra, A. (2019). Association of characteristics of psychiatrists with use of telemental health visits in the Medicare population. JAMA Psychiatry, 76(6), 654-657. https://doi. org/10.1001/jamapsychiatry.2019.0052

Chong, S. L. (2019). Making critical connections: How to apply the Analytic Guiding Frame (AGF) and Overall Guiding Frame (OGF) in qualitative data analysis. The Qualitative Report, 2, 298-306. https://nsuworks.nova.edu/tqr/vol24/iss2/.

Cheung, G., \& Chan, C. (2002). The Satir model and cultural sensitivity: A Hong Kong reflection. Contemporary Family Therapy, 24, 199-215. https://doi.org/10.1023/A:1014338025464

Cowan, K. E., McKean, A. J., Gentry, M. T., \& Hilty, D. M. (2019). Barriers to use of telepsychiatry: Clinicians as gatekeepers. Mayo Clinic Proceedings, 94(12), 2510-2523. https://doi.org/10.1016/j. mayocp.2019.04.018

D’Arrigo, P. J., Hoff, C., Knudson, M. C., \& Tuttle, A. (2017). Navigating critical theory and postmodernism: Social justice and therapist power in family therapy. Family Process, 56(3), 574-588. https:// doi.org/10.1111/famp.12236

D’Aniello, C., Nguyen, H. N., \& Piercy, F. P. (2016). Cultural sensitivity as an MFT common factor. American Journal of Family Therapy, 44(5), 234-244. https://doi.org/10.1080/01926 187.2016.1223565

Fife, S. T., D’Aniello, C., Scott, S., \& Sullivan, E. (2019). Marriage and family therapy Students' experience with common factors training. Journal of Marital and Family Therapy, 45(2), 191-205. https://doi.org/10.1111/jmft.12333

Heckathorn, D. (1997). Respondent-driven sampling: a new approach to the study of hidden populations. Social Problems, 44(2), 17499. https://doi.org/10.2307/3096941 
Ianakieva, I., Fergus, K., Ahmad, S., Pos, A., \& Pereira, A. (2016). A model of engagement promotion in a professionally facilitated online intervention for couples affected by breast cancer. Journal of Marital and Family Therapy, 42(4), 701-715. https://doi. org/10.1111/jmft.12172

Landau, J., Mittal, M., \& Weiling, E. (2008). Linking human systems: Strengthening individuals, families, and communities in the wake of mass trauma. Journal of Marital and Family Therapy, 34(2), 193-209. https://doi.org/10.1111/j.1752-0606.2008.00064.x

Langarizadeh, M., Tabatabaei, M. S., Tavakol, K., Naghipour, M., Rostami, A., \& Moghbeli, F. (2017). Telemental health care, an effective alternative to conventional mental care: A systematic Review. ActaInformaticaMedica : AIM : Journal of the Society for Medical Informatics of Bosnia \& Herzegovina : CasopisDrustvaZaMedicinskuInformatikuBiH, 25(4), 240-246. https://doi.org/10.5455/ aim.2017.25.240-246

Madigan, S., Racine, N., Cooke, J. E., \& Korczak, D. J. (2020). COVID-19 and telemental health: Benefits, challenges, and future directions. Canadian Psychology/PsychologieCanadienne. https ://doi.org/10.1037/cap0000259

Martin, J. N., Millán, F., \& Campbell, L. F. (2020). Telepsychology practice: Primer and first steps. Practice Innovations, 5(2), 114127. https://doi.org/10.1037/pri0000111

McGoldrick, M., \& Hardy, K. V. (2019). Re-Visioning family therapy : Addressing diversity in clinical practice. The Guilford Press.

Moustakas, C. (1994). Phenomenological research methods. Sage.

Pickens, J. C., Morris, N., \& Johnson, D. J. (2020). The digital divide: Couple and family therapy programs' integration of teletherapy training and education. Journal of Marital \& Family Therapy, 46(2), 186-200. https://doi.org/10.1111/jmft.12417

Perkins, S. N., Glass, V. Q., \& D'Aniello, C. (2019). It's all about the balance: Therapists' experience of systemic alliance development. Contemporary Family Therapy: An International Journal, 41(4), 420-434. https://doi.org/10.1007/s10591-019-09500-1

Pierce, B. S., Perrin, P. B., \& McDonald, S. D. (2020). Demographic, organizational, and clinical practice predictors of US psychologists' use of telepsychology. Professional Psychology: Research and Practice, 51(2), 184-193. https://doi.org/10.1037/ pro0000267

Roddy, M. K., Rothman, K., Cicila, L. N., \& Doss, B. D. (2019). Why do couples seek relationship help online? Description and comparison to in-person interventions. Journal of Marital \& Family Therapy, 45(3), 369-379. https://doi.org/10.1111/jmft.12329

Springer, P., Bischoff, R. J., Kohel, K., Taylor, N. C., \& Farero, A. (2020). Collaborative care at a distance: Student therapists' experiences of learning and delivering relationally focused telemental health. Journal of Marital and Family Therapy, 46(2), 201-217. https://doi.org/10.1111/jmft.12431

Stevenson, B. (2014). Just mercy: A story of justice and redemption. Spiegel \& Grau.

Wrape, E. R., \& McGinn, M. M. (2019). Clinical and ethical considerations for delivering couple and family therapy via telehealth. Journal of Marital and Family Therapy, 45(2), 296-308. https:// doi.org/10.1111/jmft.12319

Watson, M. F. (2019). Social justice and race in the United States: Key issues and challenges for couple and family therapy. Family Process, 58(1), 23-33. https://doi.org/10.1111/famp.12427

Publisher's Note Springer Nature remains neutral with regard to jurisdictional claims in published maps and institutional affiliations.

Valerie Q. Glass , $\mathrm{PhD}$ is a professor of Marriage and Family Therapy at Northcentral University, an online accredited MFT program. Dr. Glass is an LMFT and has a private practice.

Aurélia Bickler , $\mathrm{PhD}$ is a professor of Marriage and Family Therapy at Northcentral University, an online accredited MFT program. Dr. Bickler is an LMFT and has a private practice. 